\title{
Structure and composition of Au/Co magneto-plasmonic nanoparticles
}

\author{
Nabraj Bhattarai, Gilberto Casillas, Subarna Khanal, Daniel Bahena, and J. Jesus Velazquez-Salazar, Department of Physics and \\ Astronomy, University of Texas at San Antonio, One UTSA Circle, San Antonio, TX 78249, USA \\ Sergio Mejia, Center for Innovation and Research in Engineering and Technology, and CICFIM-Facultad de Ciencias Fisico-Matematicas, Universidad \\ Autonoma de Nuevo Leon, San Nicolas de los Garza, NL 66450, Mexico \\ Arturo Ponce, Department of Physics and Astronomy, University of Texas at San Antonio, One UTSA Circle, San Antonio, TX 78249, USA \\ Vinayak P. Dravid, Department of Material Science and Engineering, Northwestern University, 2220 Campus Drive Evanston, IL 60208, USA \\ Robert L. Whetten, Department of Physics and Astronomy, University of Texas at San Antonio, One UTSA Circle, San Antonio, TX 78249, USA \\ Marcelo M. Mariscal, INFIQC/CONICET, Departamento de Matemática y Física, Facultad de Ciencias Químicas, Universidad Nacional de Córdoba, \\ XUA5000 Córdoba, Argentina \\ Miguel Jose-Yacaman, Department of Physics and Astronomy, University of Texas at San Antonio, One UTSA Circle, San Antonio, TX 78249, USA \\ Address all correspondence to Miguel Jose-Yacaman at miguel.yacaman@utsa.edu
}

(Received 3 June 2013; accepted 7 August 2013)

\begin{abstract}
The fabrication of bimetallic magnetic nanoparticles (NPs) smaller than the size of single magnetic domain is very challenging because of the agglomeration, non-uniform size, and possible complex chemistry at nanoscale. In this paper, we present an alloyed ferromagnetic $4 \pm 1 \mathrm{~nm}$ thiolated Au/Co magnetic NPs with decahedral and icosahedral shape. The NPs were characterized by Cs-corrected scanning transmission electron microscopy (STEM) and weretheoretically studied by Grand Canonical Monte Carlo simulations. Comparison of Z-contrast imaging and energy dispersive x-ray spectroscopy used jointly with STEM simulated images from theoretical models uniquely showed an inhomogeneous alloying with minor segregation. The magnetic measurements obtained from superconducting quantum interference device magnetometer exhibited ferromagnetic behavior. This magnetic nanoalloy in the range of single domain is fully magnetized and carries significance as a promising candidate for magnetic data recording, permanent magnetization, and biomedical applications.
\end{abstract}

Magneto-plasmonic (MP) nanoparticles (NPs) are a kind of bifunctional nanoalloy or core-shell system that can be used to simultaneously enhance catalytic, magnetic, and optical properties in nanostructured systems. The core-shell NPs with magnetic cores and plasmonic shells can be used as model systems for targeted drug delivery applications, and MP NPs are currently used in biomedicine, wastewater management, magnetic resonance imaging, magnetic particle imaging, and environmental remediation. ${ }^{[1-7]}$ They can also be used in protein purification, bacterial detection, ${ }^{[8]}$ hyperthermia treatment, and cell separation. ${ }^{[6,9-11]}$ In biological applications, the use of noble-metal coatings reduces the toxicity of Co to negligible levels. When the magnetic NPs are smaller than the size of magnetic domains, the particles act as fully magnetized nanomagnets with the spins mostly in the surface, affecting both lattice constant and band structure. ${ }^{[12]}$ The absence of movement of domain walls in those smaller NPs is responsible for very high coercivity. ${ }^{[13]}$ These particles are promising materials for the construction of data storage media and high performance permanent magnetic nanocomposites, ${ }^{[14,15]}$ with high density capacity up to $1 \mathrm{~Tb} / \mathrm{in}^{2}{ }^{[16]}$

There exist several studies on the synthesis and properties of $\mathrm{Au}-\mathrm{Ni}$ and $\mathrm{Au}-\mathrm{Co} \mathrm{NPs} .{ }^{[17-23]}$ However, thiolate protected
$\mathrm{Au} / \mathrm{Co}$ NPs have not been reported so far. In general terms, segregation of $\mathrm{Au}$ toward the surface is predicted, considering only the metal-metal interaction, i.e., neglecting the effect of the surfactant molecules. Controlled synthesis is difficult, but it is known that both agglomeration and dispersion in size can be fairly controlled with an appropriate choice of capping ligands and reaction environment. ${ }^{[24]}$ Using phase transfer processes, it is possible to control the size of small NPs protected by specific thiols. ${ }^{[25,26]}$ The use of an inert gas atmosphere avoids the oxidation of Co. The detailed experimental section is presented in the supporting information (SI).

In this letter, we report ferromagnetic behavior in nanoalloyed $\mathrm{Au} / \mathrm{Co} \mathrm{NPs}$ with sizes less than $5 \mathrm{~nm}$. Imaging and chemical characterization were made using aberration corrected scanning transmission electron microscopy (STEM), which has proven to be a great technique in the characterization of metallic nanostructures. ${ }^{[27]}$ The magnetic behavior is studied using superconducting quantum interference device (SQUID) magnetometer. The STEM images are compared against simulated micrographs, calculated from the configurations obtained from grand canonical Monte Carlo (GCMC) simulations. This combination of experimental and theoretical techniques is used to study alloying and segregation at the atomic scale. 


\section{Results and discussion}

The plasmonic behavior of dodecanethiolate (DT) capped Au/ Co NP is presented in Fig. S1, where a single surface plasmon resonance peak at $507 \mathrm{~nm}$ is observed. The interaction between the incident electron beam and the thiolates present in the specimen makes the high-resolution imaging of thiolated NPs very challenging, and only in few cases they are able to resolve atomically. ${ }^{[28]}$ The high-voltage electron beam and long exposure of electron beam, even at reduced voltage might change the shape and orientation of the particles by destroying the thiols present. Those challenges were overcome by using the accelerating voltage of the electrons (in STEM) reducing to $80 \mathrm{kV}$ and heating the sample in vacuum at $60{ }^{\circ} \mathrm{C}$ (inside the microscope) overnight prior to imaging. The reduced accelerating voltage helped to reduce damage to the sample and by heating the sample in vacuum, contamination in the sample was avoided. A low magnification STEM micrograph of the Au/Co NPs is presented in Fig. 1(a) showing the uniform distribution of DT capped NPs. A representative STEM micrograph of the selected region in Fig. 1(a) is presented in Fig. 1(b), where one can clearly see the size and distribution of particles. The particles are highly monodispersed with the size ranging from 3 to $5(4 \pm 1) \mathrm{nm}$. The histogram of size distribution is presented in Fig. 1(c). The size of the NPs depends on metal (Au and Co) to thiol (sulphur) ratio during synthesis.

The morphologies mainly observed were decahedra and icosahedra (Fig. S2, SI). However, irrespective of the shape, high angle annular dark field (HAADF) STEM images, whose signal intensity depends on the atomic number, ${ }^{[29]}$ showed that the particles were nanoalloyed. Structural defects were expected since the nominal lattice mismatch between $\mathrm{Au}$ and Co is $13.8 \%$, but it appears that the small size of the particles and the presence of thiols contribute to keep the lattice relatively free of defects. In Fig. 2 left frame, the Z-contrast image shows an archetypal decahedral particle, where some of the atomic columns appear brighter than others, evidencing a relatively high presence of gold. This trend can be traced in all of the tetrahedra in the particle. The twinning angles between (111) planes varied from $69.8^{\circ}$ to $75.5^{\circ}$ as presented in Fig. 2, where a high discrepancy $\left( \pm 5^{\circ}\right)$ in region R2 and R3 is observed. The deviation of those values with the average angle $70.53^{\circ}$ between $\{111\}$ twin planes (for symmetrical monometallic decahedral nanocrystal) provides distortion and makes the particles strained. It is also possible to note shifting in some atomic columns, which provokes the elongation of bond length causing internal lattice strain; however, the small size of the nanostructure avoids the appearance of partial dislocations. Gold atoms are persistently present in the adjacent columns of twin boundary planes and this presence decreases far from the twinning plane. The intensity line profile in the Z-contrast image is measured along the dotted black arrow as represented by 1,2 , and 3 and is presented in the right frame in Fig. 2. The stronger intensity in the profile corresponds to the Au-rich atomic columns and the weaker intensity corresponds to the Co-rich atomic columns. Profiles 1 and 3 show the diffusion and intermixing of $\mathrm{Au}$ and $\mathrm{Co}$ forming an alloyed structure. Similar patterns can be observed in other atomic planes, which indicate some segregation.

The elemental distribution between $\mathrm{Au}$ and $\mathrm{Co}$ atoms was confirmed using energy dispersive x-ray spectroscopy (EDS). Representative EDS line profile and mapping are presented in Fig. 3. The EDS line spectra of [Fig. 3(b)] were measured over the line drawn in Fig. 3(a); the red and green line in the plot correspond to the contribution from Au-L $(9.71 \mathrm{keV})$ and $\mathrm{Au}-\mathrm{M}(2.12 \mathrm{keV})$, while the blue line is the contribution from Co-L $(0.77 \mathrm{keV})$. Area EDS maps taken over a whole particle are presented in Figs. 3(c)-3(g). Both line profile and map spectra confirmed the mixing of Au and Co forming an alloyed structure.

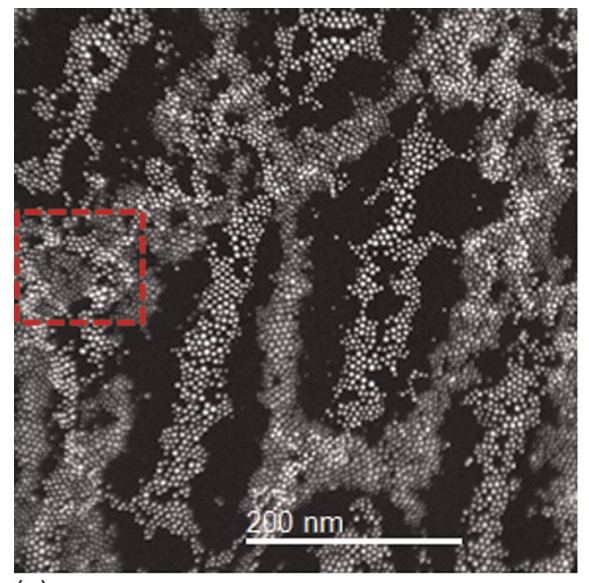

(a)

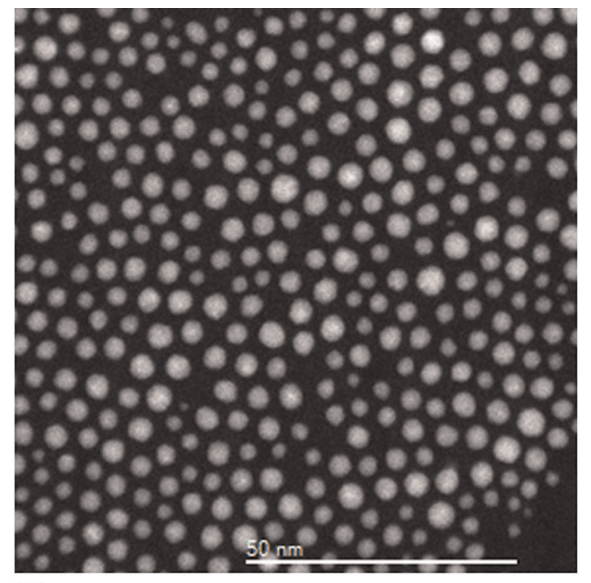

(b)

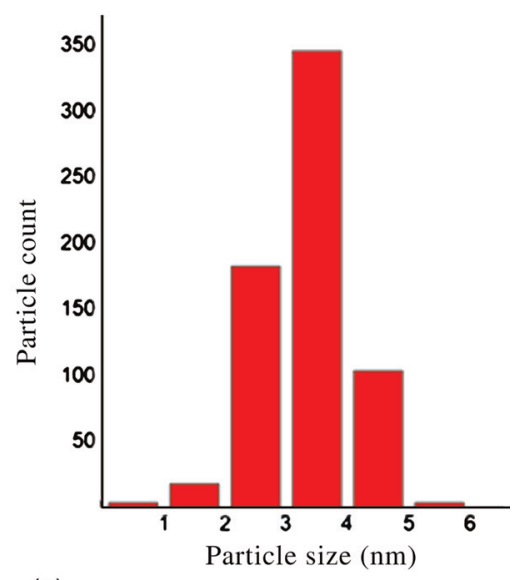

(c)

Figure 1. Low magnification STEM images of Au/Co NPs in amorphous carbon. (a) Large coverage particle distribution in amorphous carbon grid. (b) Magnified portion of selected area [red dotted square in (a)] showing the uniform monolayer film of NPs. (c) Particle size histogram, showing a narrow distribution around $2.5-5 \mathrm{~nm}$. 

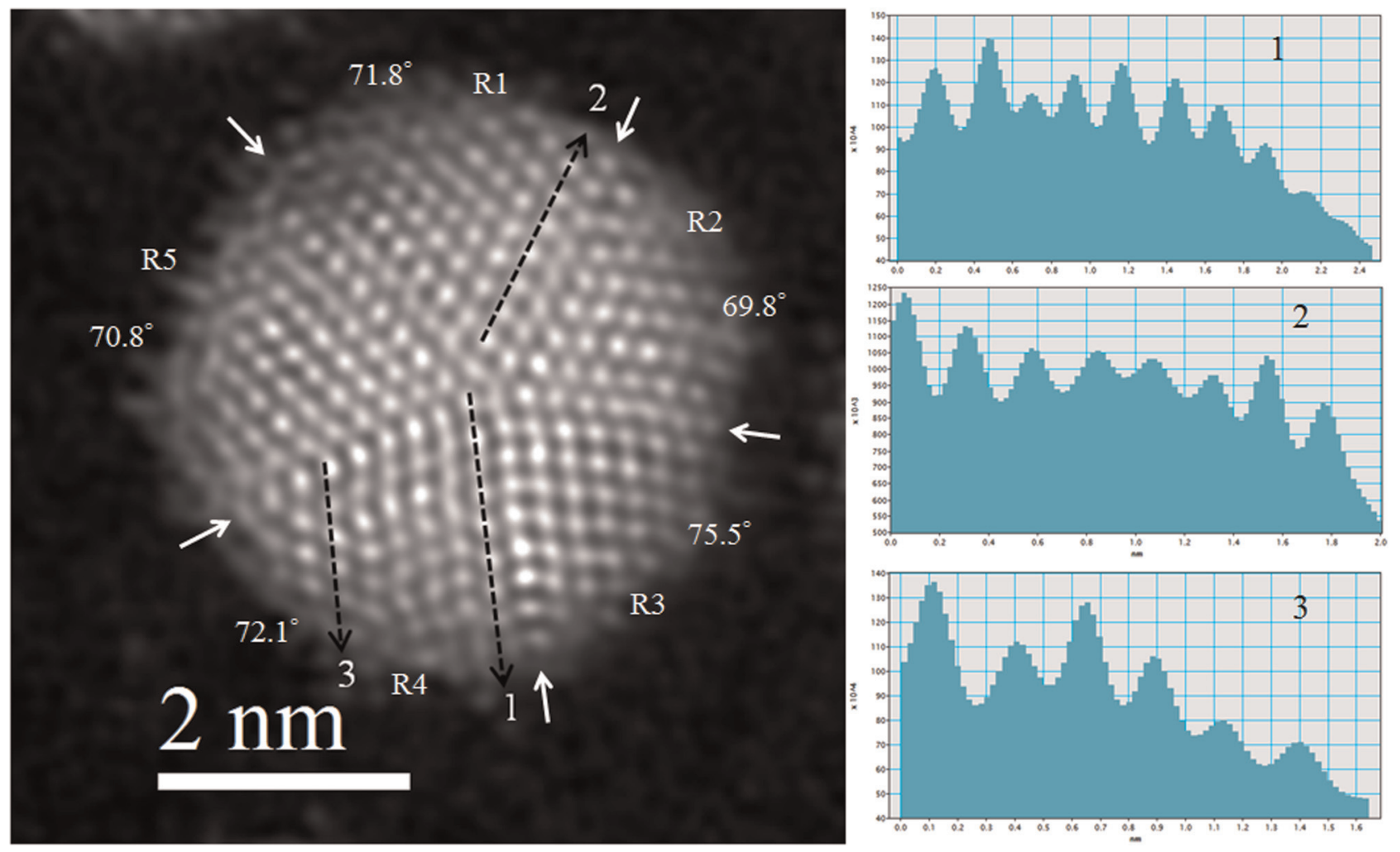

Figure 2. Left, a magnified HAADF-STEM image of decahedral shaped Au/Co NPs along [110] zone axis. Each tetrahedron (R1, R2, R3, R4, and R5) is identified by white arrow and the angles measured between the $\{111\}$ twinning plane are $71.8^{\circ}, 69.8^{\circ}, 75.5^{\circ}, 72.1^{\circ}$, and $70.8^{\circ}$ as indicated. The intensity profile is measured along different lattice planes in the decahedral structure. The profile is drawn from the center to outwards as indicated by dotted arrows 1,2 , and 3. Each profile is represented as 1,2, and 3 and presented in the plot in the frame in right. The stronger intensity in the profile corresponds to Au-rich atomic columns and the weaker intensity corresponds to Co-rich atomic columns. Profiles 1 and 3 show the diffusion and intermixing of Au and Co forming an alloyed structure.

In order to understand the growing sequence of $\mathrm{Co} / \mathrm{Au} \mathrm{NPs}$, we implemented a set of GCMC simulations, ${ }^{[30-33]}$ using decahedra gold clusters as seeds, as Au nuclei may be formed earlier than Co. ${ }^{[34]}$ For each simulation run, $1^{\prime} 10^{5} \mathrm{MC}$ steps were used to equilibrate the structures at $300 \mathrm{~K}$, and Co atoms were added to the system at fixed chemical potential. Fig. S3 in SI represents several structures at several growth stages. In Fig. S2(a), the initial $\mathrm{Au}^{\mathrm{Dh}}$ seed after relaxation is shown. Figures S2(b)- S2(d) show the Co nucleation at different coverage degrees $q$. It is worth noting that the deposition of Co atoms starts with the formation of surface alloy (i.e., Co-Au intermixing) instead of a layer-by-layer fashion. This can be understood in terms of the surface energy $(\gamma)$ of both metals. The surface energy of $\mathrm{Co} \gamma_{\mathrm{hcp}(0001)}^{\mathrm{Co}}=2.11 \mathrm{~J} / \mathrm{m}^{2},{ }^{[35]}$ whereas for $\mathrm{Au}$, $\gamma_{\mathrm{fcc}(111)}^{\mathrm{Au}}=0.707 \mathrm{~J} / \mathrm{m}^{2},{ }^{[36]}$ from pure energetic considerations, Co will exchange with $\mathrm{Au}$ atoms. The activation energies for these exchange processes is of the order of $0.11 \mathrm{eV}^{[18]}$ Therefore, from the kinetic considerations the exchange between $\mathrm{Co}$ and $\mathrm{Au}$ is also favorable. Since $E_{\mathrm{bind}}^{\mathrm{Co}-\mathrm{Co}}>E_{\mathrm{bind}}^{\mathrm{Au}-\mathrm{Co}}, 3 \mathrm{D}$ phase formation takes place regardless of the $\mathrm{Co}-\mathrm{Au}$ misfit according to the Volmer-Weber growth mechanism in the over-potential range. ${ }^{[37]}$ A closer inspection of the atomic distribution of each element reveals the diffusion of Co atoms to intermediate layers, which is in excellent agreement with previous reports on Co deposition on $\mathrm{Au}(111)$ surfaces, ${ }^{[38]}$ and with predictions in bimetallic AuCo particles, where the tendency of Co is to populate sites close but not on the surface of the particle. ${ }^{[39]}$

The atomic configurations shown in Figs. S2(b) and S2(c) (decahedra with $q=0.25 \mathrm{ML}$ and $q=1.03 \mathrm{ML}$, respectively) were used as input to simulate HAADF-STEM images. ${ }^{[40]}$ The simulated micrographs are presented in Figs. 4(c) and 4 (d) and compared against the experimental images in Figs. 4 (a) and 4(b). Remarkably, all of the simulated micrographs resemble to those obtained experimentally, even at the smallest sizes. This is particularly interesting considering that the GCMC simulations included metal-metal interactions. With respect to the effect of the surfactant molecules (i.e., RS, R= alkyl group) on the composition of the AuCo NCs, theoretical calculations, using density functional theory, predict an adsorption energy of alkylthiolates on the $\mathrm{Au}(111)$ surface ranging from -1.14 to $-2.22 \mathrm{eV}$ depending on the adsorption site, ${ }^{[41]}$ whereas the adsorption energy of RS on $\mathrm{Co}(0001)$ surface was reported as $-3.0 \mathrm{eV},{ }^{[42]}$ which means that the 


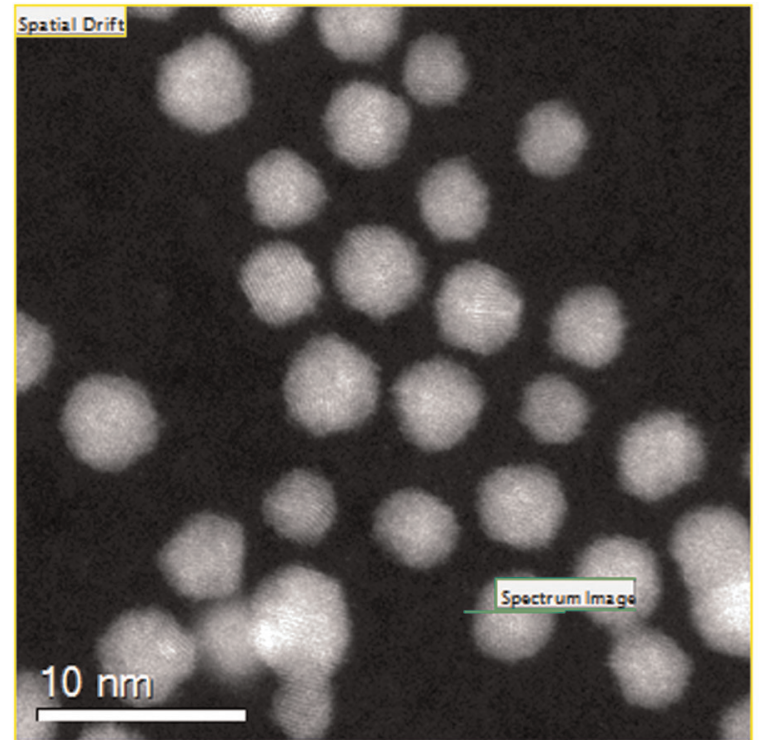

(a)

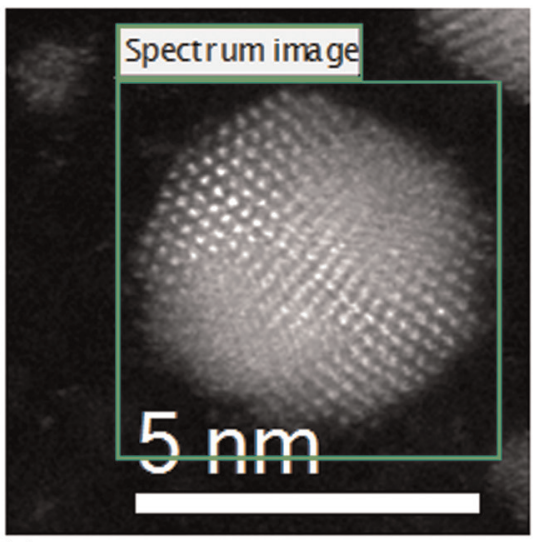

(c)

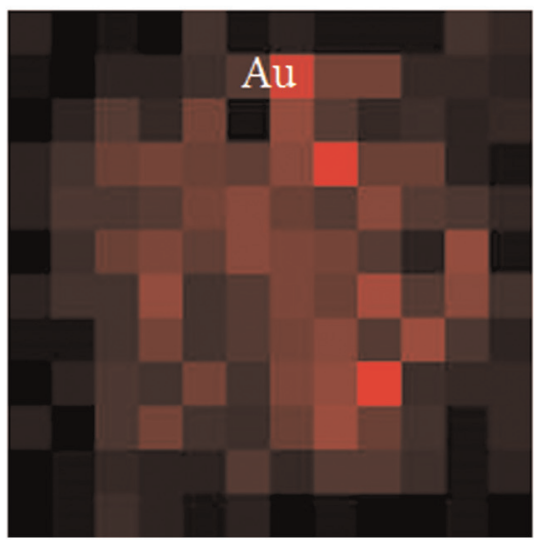

(f)

(d)

(g)

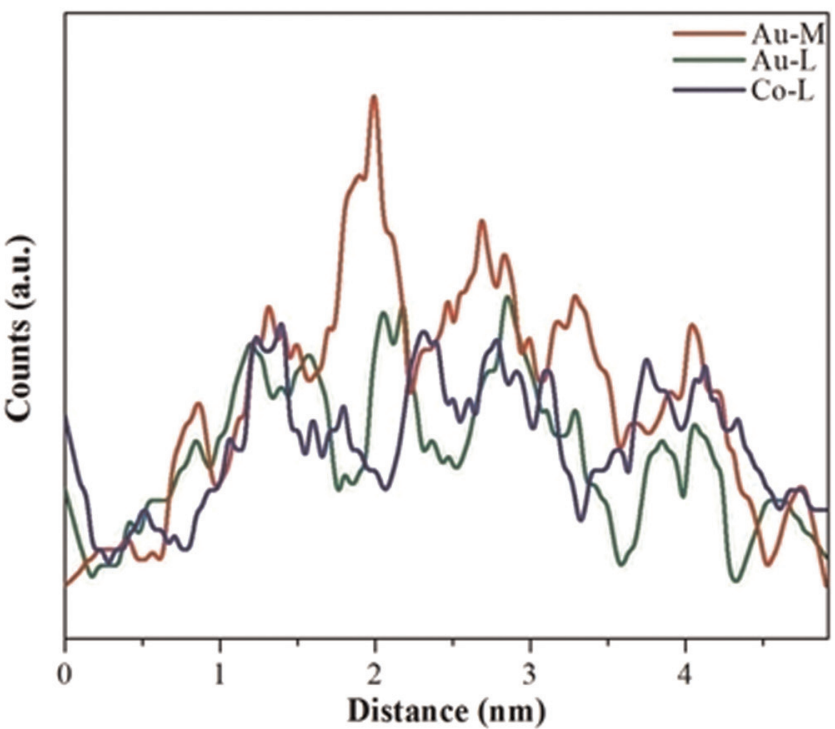

(b)
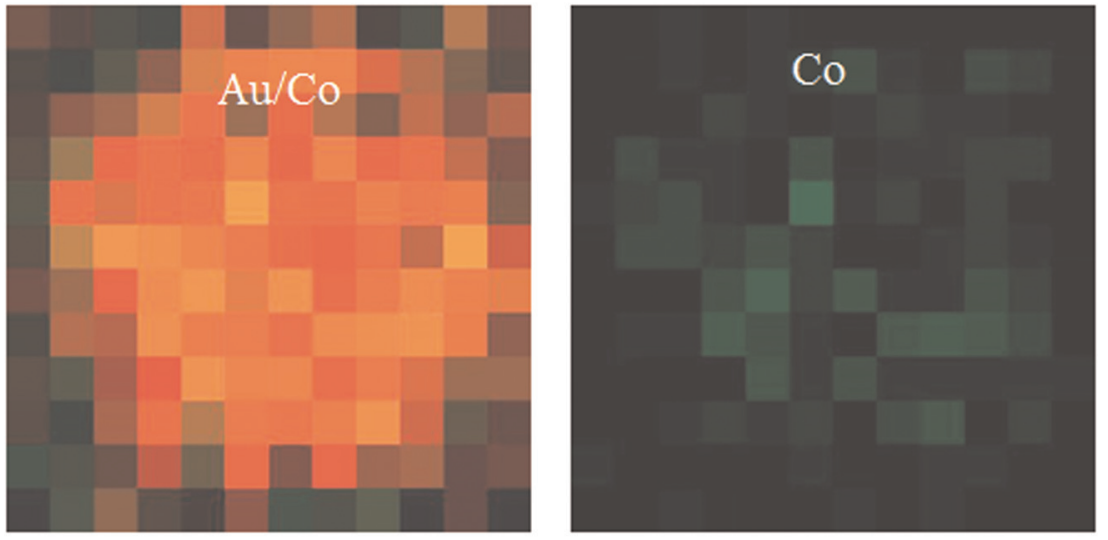

(e)

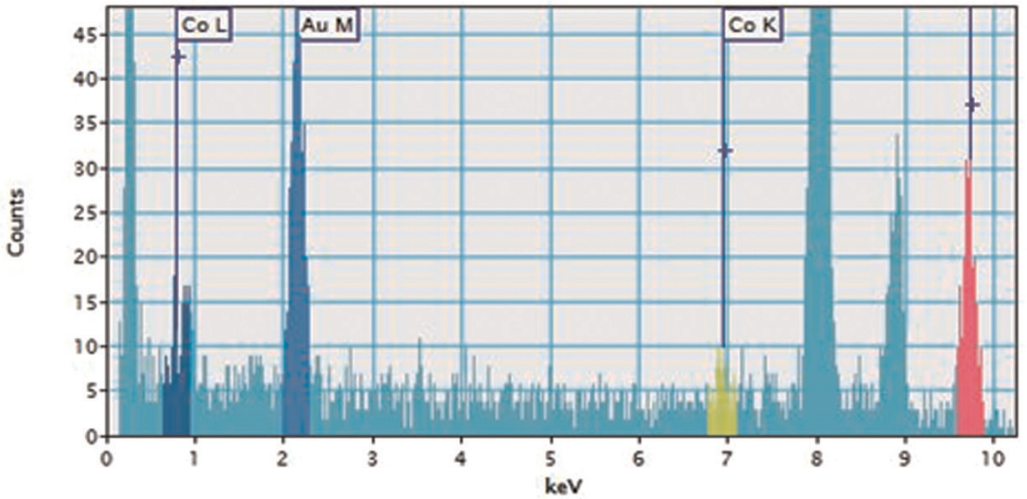

Figure 3. EDS line profile and mapping of single Au/Co nanocluster. (a) Dark field images with the spatial drift and spectrum image during recording EDS spectra. (b) The line profile spectrum (number of counts in arbitrary unit versus position in $\mathrm{nm}$ ) obtained during EDS line scanning for the spectrum image in figure a. (c) Au/Co sample for EDS mapping and (d) Au/Co mapping from L-shell. (e) and (f) Co and Au mapping from L-shell and (g) Line spectrum of different elements present in the sample. The signal contributions from Au-M, Au-L, and $\mathrm{Co}-\mathrm{L}$ are represented by red, green, and blue spectra as inset in figure (b). The intensity recorded at different positions of $\mathrm{Au}$ and $\mathrm{Co}$ gives intermixing and confirms the alloyed structure. 


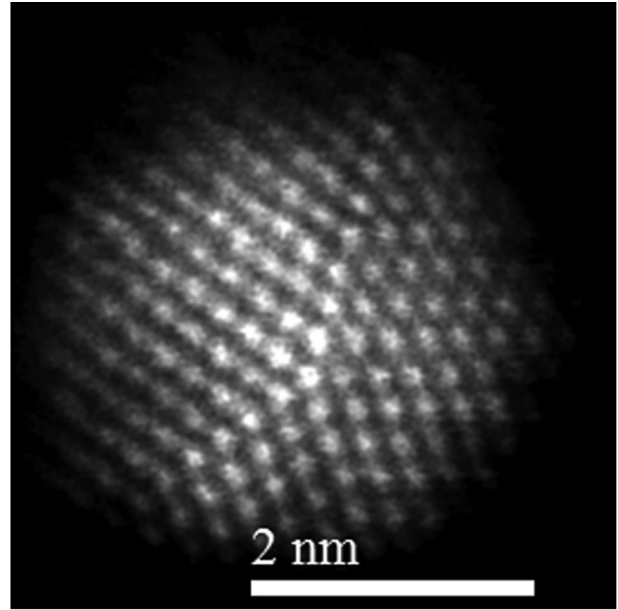

(a)

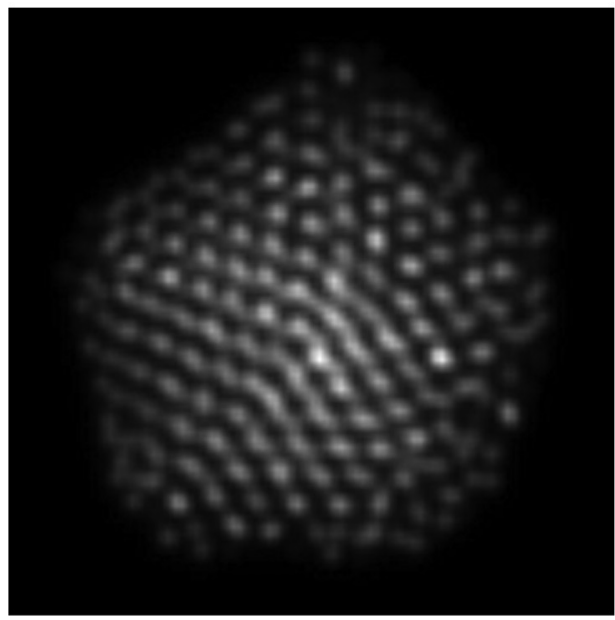

(c)

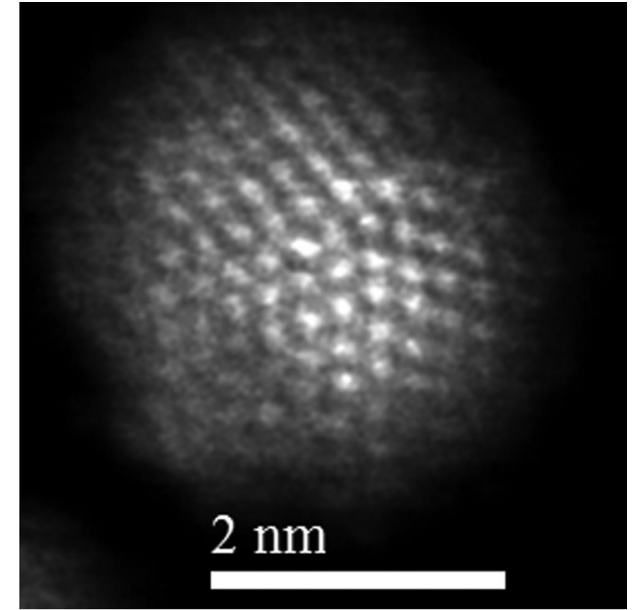

(b)

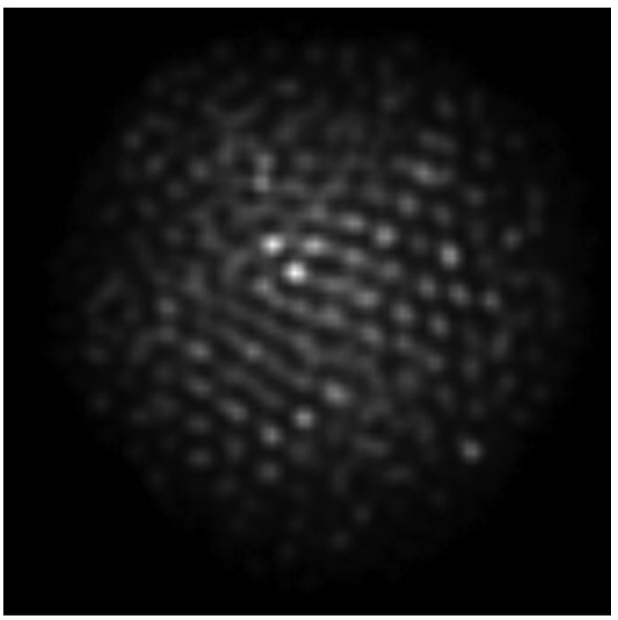

(d)

Figure 4. Comparison between experimental and simulated STEM images. (a) and (b) experimental HAADF-STEM images. (c) and (d) STEM simulations taken from the configurations shown in Fig. $4(\mathrm{~b})(\theta=0.25 \mathrm{ML})$ and Fig. 4(c) $(\theta=1.03 \mathrm{ML})$ respectively.
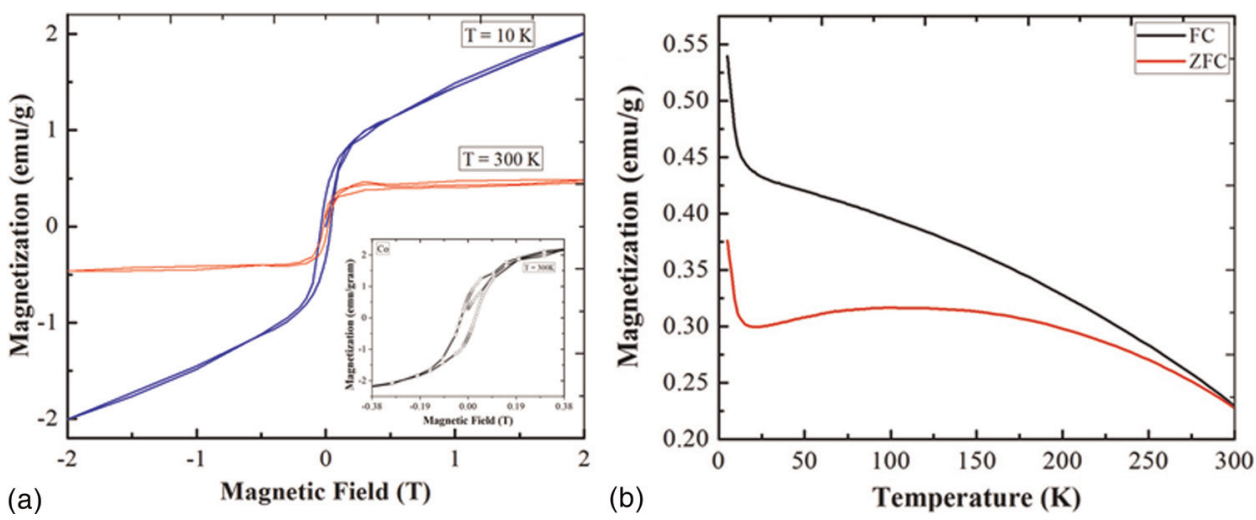

Figure 5. Magnetic properties measurement for Au/Co NPs by using SQUID magnetometer. (a) Hysteresis loop for magnetization (emu/g) versus magnetizing field ( $\mathrm{T}$, tesla) measurements at room temperature (red) and low temperature $10 \mathrm{~K}$ (blue). The inset represents the magnified portion in the center of the histogram at room temperature for 1-3 nm sized Co NPs. (b) Magnetization (emu/g) as a function of temperature (K). Upper curve: FC, field cooled; lower curve: ZFC, zero field cooled. 
interaction between the thiolate molecules with Co is much stronger than with $\mathrm{Au}$. Therefore, Co segregation toward the surface of the NP is favored.

The magnetic property of Au/Co NP (powder sample) was investigated by SQUID magnetometry. The hysteresis loops recorded at 300 and $10 \mathrm{~K}$ are presented in Fig. 5(a), where the change in magnetization is observed by changing the strength of the magnetizing field from -2 to 2 Tesla. The coercive field was $346 \mathrm{Oe}$ at $300 \mathrm{~K}$ and $796 \mathrm{Oe}$ at $10 \mathrm{~K}$, while the retentive magnetizations were 0.257 and $0.638 \mathrm{emu} / \mathrm{g}$. The saturation magnetization value was $0.472 \mathrm{emu} / \mathrm{g}$ at $300 \mathrm{~K}$. The effective magnetic moment was obtained by fitting a Langevin function in units of Bohr's magneton $\left(\mu_{B}\right)$ at low temperature, and found to be $2.8 \times 10^{2} \mu_{\mathrm{B}}$. Taking the case of low magnetic field (200 gauss), the magnetic moment at room temperature can be estimated to be $1.1429 \times 10^{4} \mu_{\mathrm{B}}$. The hysteresis loop for monometallic $1-3 \mathrm{~nm}$ sized Co NPs synthesized in the same way as $\mathrm{Au} / \mathrm{Co} \mathrm{NP}$ is presented in the inset of Fig. 5a, where both coercive and retentive values are found to be higher than bimetallic $(\mathrm{Au} / \mathrm{Co})$ case. Figure $5 \mathrm{~b}$ shows the temperature dependent magnetization measurements for field cooled and zero field cooled of Au/Co NPs. Despite very small particles as reported by Kechrakos ${ }^{[43]}$ et al. and Dormann ${ }^{[44]}$ et al. Au/Co NPs present ferromagnetic behavior with coercive field and retentive magnetization.

In a 2008 study, Auten et al. ${ }^{[23]}$ have reported "3-nm magnetic NiAu nanoparticles" using decane-thiolate monolayer protection, which "had essentially the same $\mathrm{Au}: \mathrm{Ni}$ ratio as was set in the synthesis (Au:Ni=2.1)." The evidence indicated the surfaces are enriched in gold. The saturation magnetization was $\sim 10 \mathrm{emu} / \mathrm{g}-\mathrm{Ni}$, as compared $\sim 0.5 \mathrm{emu} / \mathrm{g}$-total in the present case.

\section{Summary}

In summary, this study reveals the formation of magnetic nanoalloy in $\mathrm{Au} / \mathrm{Co}$ NPs within the magnetic domain size. Atomically resolved HAADF-STEM images obtained from aberration-corrected STEM are allowed to investigate the elemental distribution in individual atomic columns in the $\mathrm{NPs}$, revealing the diffusion of $\mathrm{Co}$ atoms into the Au lattice site. This segregation and the nanoalloy of $\mathrm{Au}$ and $\mathrm{Co}$ were unequivocally supported by EDS mapping and line profile measurements. The experimental results were also supported and interpreted by the GCMC simulations; in particular, the atomistic simulations predict $\mathrm{Au}-\mathrm{Co}$ mixing at the early stages of the growth process and the so-called bridging phenomenon $(\mathrm{Au}-\mathrm{Co} \mathrm{Au}-\mathrm{Au})$ at coverage degrees close to unity. The magnetic properties obtained from the SQUID magnetometer showed that the particles have a ferromagnetic behavior, that will be very promising applications in high-density information storage, possible permanent magnetic nanocomposites. Moreover, MP 3-5 nm sized Au/Co NPs are below the magnetic domain size range and behaves as the fully magnetized nanomagnet with a large number of spins in the surface, which is responsible for important magnetic contribution and also a possible candidate for biomedical applications where the toxicity can be reduced by reducing the concentration of $\mathrm{Co}$ in the $\mathrm{Au} / \mathrm{Co}$ system.

\section{Supplementary materials}

For supplementary material for this article, please visit http:// dx.doi.org/10.1557/mrc.2013.30

\section{Acknowledgments}

This project was supported by grants from the National Center for Research Resources (5 G12RR013646-12) and the National Institute on Minority Health and Health Disparities (G12MD007591) from the National Institutes of Health. The authors would like to acknowledge the NSF for support with grants DMR-1103730, "Alloys at the Nanoscale: The Case of Nanoparticles Second Phase and PREM: NSF PREM Grant \# DMR 0934218; "Oxide and Metal Nanoparticles- The Interface Between Life Sciences and Physical Sciences". NB acknowledges G. Ajithkumar for discussion about magnetic behavior. Support from the Mexican Council for Science and Technology (CONACYT, Mexico), through project CIAM 148967, is also acknowledged. MMM wish to thank CONICET, SeCyT UNC, ANPCyT Program BID (PICT 2010-123), and PIP: 112-200801-000983 "Nanotechnology in-silico" for financial support.

\section{References}

1. A.-H. Lu, W. Schmidt, N. Matoussevitch, H. Bönnemann, B. Spliethoff, B. Tesche, E. Bill, W. Kiefer, and F. Schüth: Nanoengineering of a magnetically separable hydrogenation catalyst. Angew. Chem., Int. Ed. 43, 4303 (2004).

2. G. Reiss and A. Hutten: Magnetic nanoparticles: applications beyond data storage. Nat. Mater. 4, 725 (2005).

3. B. Gleich and J. Weizenecker: Tomographic imaging using the nonlinear response of magnetic particles. Nature 435, 1214 (2005).

4. R.N. Grass, E.K. Athanassiou, and W.J. Stark: Covalently functionalized cobalt nanoparticles as a platform for magnetic separations in organic synthesis. Angew. Chem., Int. Ed. 46, 4909 (2007).

5. D.W. Elliott and W.X. Zhang: Field assessment of nanoscale bimetallic particles for groundwater treatment. Environ. Sci. Technol. 35, 4922 (2001).

6. J. Dobson: Magnetic nanoparticles for drug delivery. Drug Dev. Res. 67, 55 (2006).

7. Y. Kobayashi, M. Horie, M. Konno, B. Rodríguez-González, and L. M. Liz-Marzán: Preparation and properties of silica-coated cobalt nanoparticlest. J. Phys. Chem. B 107, 7420 (2003).

8. J. Gao, H. Gu, and B. Xu: Multifunctional magnetic nanoparticles: design, synthesis, and biomedical applications. Acc. Chem. Res. 42, 1097 (2009).

9. Y. Bao and K.M. Krishnan: Preparation of functionalized and gold-coated cobalt nanocrystals for biomedical applications. J. Magn. Magn. Mater. 293, 15 (2005).

10. Q.A. Pankhurst, J. Connolly, S.K. Jones, and J. Dobson: Applications of magnetic nanoparticles in biomedicine. J. Phys. D: Appl. Phys. 36, R167 (2003).

11.Z. Lu, M.D. Prouty, Z. Guo, V.O. Golub, C.S.S.R. Kumar, and Y.M. Lvov: Magnetic switch of permeability for polyelectrolyte microcapsules embedded with Co@Au nanoparticles. Langmuir 21, 2042 (2005).

12. A.H. Lu, E.e.L. Salabas, and F. Schüth: Magnetic nanoparticles: synthesis, protection, functionalization, and application. Angew. Chem., Int. Ed. 46, 1222 (2007). 
13. T. Iwaki, Y. Kakihara, T. Toda, M. Abdullah, and K. Okuyama: Preparation of high coercivity magnetic FePt nanoparticles by liquid process. J. App. Phys. 94, 6807 (2003).

14. M. Chen, J. Kim, J.P. Liu, H. Fan, and S. Sun: Synthesis of FePt nanocubes and their oriented self-assembly. J. Am. Chem. Soc. 128, 7132 (2006).

15. S. Sun, C. Murray, D. Weller, L. Folks, and A. Moser: Monodisperse FePt nanoparticles and ferromagnetic FePt nanocrystal superlattices. Science 287, 1989 (2000).

16.D. Weller and A. Moser: Thermal effect limits in ultrahigh-density magnetic recording. IEEE Trans. Magn. 35, 4423 (1999).

17. A. Rapallo, J. Olmos-Asar, O. Oviedo, M. Ludueña, R. Ferrando, and M. Mariscal: Thermal properties of Co/Au nanoalloys and comparison of different computer simulation techniques. J. Phys. Chem. C 116, 17210 (2012).

18. O. Oviedo, E. Leiva, and M. Mariscal: Diffusion mechanisms taking place at the early stages of cobalt deposition on Au (111). J. Phys.: Condens. Matter. 20, 265010 (2008).

19. A. Mayoral, S. Mejia-Rosales, M.M. Mariscal, E. Perez-Tijerina, and M. Jose-Yacaman: The Co-Au interface in bimetallic nanoparticles: a high resolution STEM study. Nanoscale 2, 2647 (2010).

20. F. Bao, J.-F. Li, B. Ren, Jian-Lin Yao R.-A. Guand Z.-Q. Tian: Synthesis and characterization of Au@ Co and Au@ Ni core-shell nanoparticles and their applications in surface-enhanced Raman Spectroscopy. J. Phys. Chem. C 112, 345 (2008).

21. Y. Bao, H. Calderon, and K.M. Krishnan: Synthesis and characterization of magnetic-optical Co-Au core-shell nanoparticles. J. Phys. Chem. C 111, 1941 (2007).

22.D. Wang and Y. Li: One-pot protocol for Au-based hybrid magnetic nanostructures via a noble-metal-induced reduction process. J. Am. Chem. Soc. 132, 6280 (2010).

23.B.J. Auten, B.P. Hahn, G. Vijayaraghavan, K.J. Stevenson and B.D. Chandler: Preperation and Characterization of $3 \mathrm{~nm}$ Magnetic NiAu Nanoparticles. J. Phys. Chem. C 112, 5365 (2008).

24. M. Mariscal, J. Olmos-Asar, C. Gutierrez-Wing, A. Mayoral, and M. Yacaman: On the atomic structure of thiol-protected gold nanoparticles: a combined experimental and theoretical study. Phys. Chem. Chem. Phys. 12, 11785 (2010).

25. A. Frenkel, S. Nemzer, I. Pister, L. Soussan, T. Harris, Y. Sun, and M. Rafailovich: Size-controlled synthesis and characterization of thiol-stabilized gold nanoparticles. J. Chem. Phys. 123, 184701 (2005).

26. M. Brust, D.J. Schiffrin, D. Bethell, and C.J. Kiely: Novel gold-dithiol nanonetworks with non-metallic electronic properties. Adv. Mater. 7, 795 (1995).

27.N. Bhattarai, G. Casillas, S. Khanal, J.J.V. Salazar, A. Ponce, and M. Jose-Yacaman: Origin and shape evolution of core-shell nanoparticles in $\mathrm{Au}-\mathrm{Pd}$ : from few atoms to high Miller index facets. J. Nanopart. Res. 15, 1 (2013).

28. D. Bahena, N. Bhattarai, U. Santiago, A. Tlahuice, A. Ponce, S.B.H. Bach, B. Yoon, R.L. Whetten, U. Landman, and M. Jose-Yacaman: STEM electron diffraction and high-resolution images used in the determination of the crystal structure of the Au144(SR)60 cluster. J. Phys. Chem. Lett. 4, 975 (2013)

29. S. Pennycook: Z-contrast STEM for materials science. Ultramicroscopy 30, 58 (1989).

30. M.M. Mariscal, J.J. Velázquez-Salazar, and M.J. Yacaman: Growth mechanism of nanoparticles: theoretical calculations and experimental results. CrystEngComm 14, 544 (2012).

31. M. Mariscal, 0. Oviedo, and E. Leiva: On the selection of facets in metallic nanoparticles. J. Mater. Res. 27, 1777 (2012).

32. F. Cleri and V. Rosato: Tight-binding potentials for transition metals and alloys. Phy. Rev. B 48, 22 (1993).

33.D. Frankel and B. Smith: Understanding Molecular Simulation: From Algorithms to Applications (Academic Press, San Diego, CA, 1996).

34. D.R. Lide: CRC Handbook of Chemistry and Physics: A Ready-Reference Book of Chemical and Physical Data (CRC Press, Boca Raton, FL, 1999).

35. M.P. Punkkinen, Q.-M. Hu, S.K. Kwon, B. Johansson, J. Kollár, and L. Vitos: Surface properties of $3 \mathrm{~d}$ transition metals. Philos. Mag. 91, 3627 (2011).
36. V. Zólyomi, L. Vitos, S. Kwon, and J. Kollár: Surface relaxation and stress for $5 d$ transition metals. J. Phys.: Condens. Matter. 21, 095007 (2009).

37. W. Lorenz and G. Staikov: 2D and 3D thin film formation and growth mechanisms in metal electrocrystallization -an atomistic view by in situ STM. Surf. Sci. 335, 32 (1995).

38. H. Guo, J. Li and B. Liu: Atomistic modeling and thermodynamic interpretation of the bridging phenomenon observed in the Co-Au system. Phy. Rev. B 70, 195434 (2004)

39. D. Bochicchio and R. Ferrando: Morphological instability of core-shell metallic nanoparticles. Phy. Rev. B 87, 165435 (2013).

40. K. Ishizuka: A practical approach for STEM image simulation based on the FFT multislice method. Ultramicroscopy 90, 71 (2002).

41. J.A. Olmos-Asar, A. Rapallo, and M.M. Mariscal: Development of a semiempirical potential for simulations of thiol-gold interfaces. Application to thiol-protected gold nanoparticles. Phys. Chem. Chem. Phys. 13, 6500 (2011).

42. A. Caruso, L. Wang, S. Jaswal, E.Y. Tsymbal, and P.A. Dowben: The interface electronic structure of thiol terminated molecules on cobalt and gold surfaces. J. Mater. Sci. 41, 6198 (2006).

43. D. Kechrakos and K.N. Trohidou: Magnetic properties of dipolar interacting single-domain particles. Phy. Rev. B 58, 12169 (1998).

44.J.L. Dormann, D. Fiorani, and E. Tronc: Magnetic relaxation in fineparticle systems. Adv. Chem. Phys. 283 (2007) 